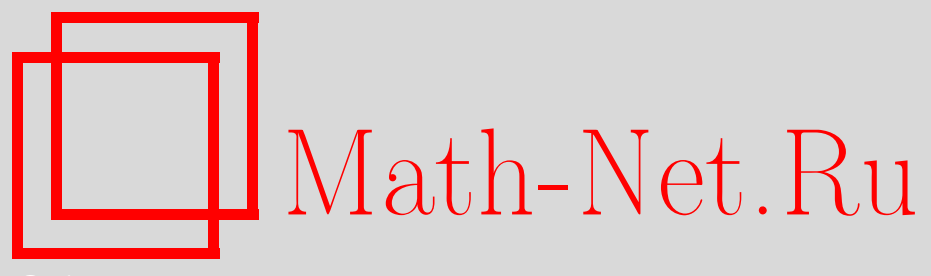

А. А. Карацуба, Об одном свойстве множества простых чисел, УМН, 2011, том 66, выпуск 2, 3-14

DOI: https://doi.org/10.4213/rm9419

Использование Общероссийского математического портала Math-Net.Ru подразумевает, что вы прочитали и согласны с пользовательским соглашением http://www . mathnet.ru/rus/agreement

Параметры загрузки:

IP: 3.80 .253 .173

26 апреля 2023 г., 06:57:25

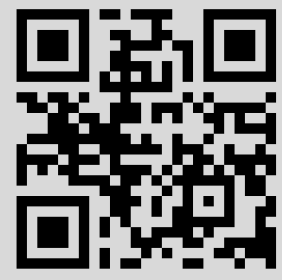




\section{Об одном свойстве множества простых чисел}

\section{А. А. Карацуба}

Обнаружен эффект взаимозависимости структуры чисел натурального ряда и вида простых сомножителей, составляющих эти числа. Статья подготовлена к печати Е. А. Карацубой и М. Е. Чангой по черновикам и заметкам А. А. Карацубы 2007-2008 гг., детальные вычисления выполнены М. Е. Чангой.

Библиография: 10 названий.

Ключевые слова: простые делители, арифметические прогрессии.

1. Введение. Пусть $\mathbb{N}$ есть множество натуральных чисел, т. е. числа $1,2,3, \ldots$. Множество простых чисел, т. е. таких $n \in \mathbb{N}, n>1$, которые имеют ровно два разных делителя (именно, $n$ и 1 ). Будем обозначать $\mathbb{P}, \mathbb{P}=$ $\{2,3,5,7,11, \ldots\} \subset \mathbb{N}$.

Простые числа занимают особое место среди натуральных чисел, являясь мультипликативным базисом натурального ряда. Это означает, что каждое $n \in \mathbb{N}, n>1$, представляется в виде произведения простых чисел (не обязательно различных), т. е. $n=p_{1} \cdots p_{k}$, где $p_{1} \in \mathbb{P}, \ldots, p_{k} \in \mathbb{P}$, и такое представление единственно с точностью до порядка сомножителей.

Если рассмотреть числа, имеющие четное и соответственно нечетное количество простых сомножителей в их каноническом представлении, то количество тех и других среди всех чисел натурального ряда приблизительно одинаковое.

Если рассмотреть те же числа, но при условии, что в их каноническое представление не входят простые числа, принадлежащие какой-либо арифметической прогрессии, скажем, прогрессии $6 m+1, m=1,2, \ldots$, или прогрессии $k m+l, 1 \leqslant l<k,(l, k)=1, m=0,1,2, \ldots$, то картина будет иной: чисел с четным количеством простых сомножителей будет меньше, чем с нечетным количеством простых сомножителей.

Этому и похожим эффектам посвящена настоящая статья.

2. Обозначения. Далее используются следующие обозначения: символом $(a, b)$ обозначается наибольший общий делитель чисел $a$ и $b$; запись $d \mid n$ при натуральном $d$ и целом $n$ означает, что $d$ делит $n$; символами $p, p_{1}, p_{2}, \ldots$ обозначаются простые числа, символом $s=\sigma+i t$ обозначается комплексное переменное; $\varphi(k)$ - функция Эйлера (количество натуральных чисел, не превосходящих $k$ 
и взаимно простых с $k) ; \mu(n)$ - функция Мёбиуса:

$$
\mu(n)= \begin{cases}1, & \text { если } n=1, \\ 0, & \text { если } n=p^{2} m, \\ (-1)^{k}, & \text { если } n=p_{1} \cdots p_{k} ;\end{cases}
$$

характер Дирихле $\chi(a)$ по модулю $m$ - это любая функция, определенная для всех целых $a$ и удовлетворяющая условиям:

а) $\chi(a)=0$, если $(a, m)>1$,

б) $\chi(a)$ не равна нулю тождественно,

в) $\chi\left(a_{1} a_{2}\right)=\chi\left(a_{1}\right) \chi\left(a_{2}\right)$,

г) $\chi(a+m)=\chi(a)$;

$\zeta(s)$ - дзета-функция Римана; запись $A \ll B$ означает, что $|A| \leqslant c B$; запись $A \asymp B$ с $B>0$ означает, что $c_{1} B \leqslant A \leqslant c_{2} B ; \pi(x)$ - количество простых чисел, не превосходящих $x$; при $\operatorname{Re} s>1$ определим функцию $\ln L(s, \chi)$ соотношением

$$
\ln L(s, \chi)=\sum_{p} \sum_{m=1}^{\infty} \frac{\chi^{m}(p)}{m p^{m s}} ;
$$

$c, c_{0}, c_{1}, \ldots, C, C_{0}, C_{1}, \ldots$ - положительные абсолютные постоянные, в разных утверждениях, вообще говоря, разные; постоянные в знаках $O$ являются абсолютными, если это не оговаривается особо.

3. Асимптотики Ландау. Пусть $\mathbb{N}_{0}$ и $\mathbb{N}_{1}$ - подмножества $\mathbb{N}$ такие, что $n \in \mathbb{N}_{0}$, если $n$ содержит четное число простых сомножителей, и $n \in \mathbb{N}_{1}$, если $n$ содержит нечетное число простых сомножителей. Интуитивно ясно, что количество чисел в $\mathbb{N}_{0}$ и $\mathbb{N}_{1}$ приблизительно одинаково. Чтобы сформулировать последнее утверждение математически точно, введем при $x \geqslant 1$ две функции $n_{0}(x)$ и $n_{1}(x)$, где $n_{0}(x)$ - количество чисел $n$ из $\mathbb{N}_{0}$ таких, что $n \leqslant x$, а $n_{1}(x)-$ количество чисел $n$ из $\mathbb{N}_{1}$ таких, что $n \leqslant x$.

Асимптотические формулы для $n_{0}(x)$ и $n_{1}(x)$ были получены Э. Ландау в [1] (см. также [2]) методом комплексного интегрирования:

$$
\begin{aligned}
& n_{0}(x)=\frac{1}{2} x+O\left(x e^{-c \sqrt{\ln x}}\right), \\
& n_{1}(x)=\frac{1}{2} x+O\left(x e^{-c \sqrt{\ln x}}\right) .
\end{aligned}
$$

Они показывают, что

$$
n_{0}(x) \sim n_{1}(x) \sim \frac{1}{2} x
$$

т. е. $n_{0}(x)$ и $n_{1}(x)$ асимптотически равны. Кроме того,

$$
n_{1}(x)-n_{0}(x)=O\left(x e^{-c \sqrt{\ln x}}\right),
$$

т. е. эта разность мала. Заметим также, что в [1] доказана самая общая теорема об асимптотической формуле сумматорной функции коэффициентов ряда Дирихле (см. также [3; гл. 7, п. 49]). 


\section{Namay [] Dokaya per efunx Terpen o}

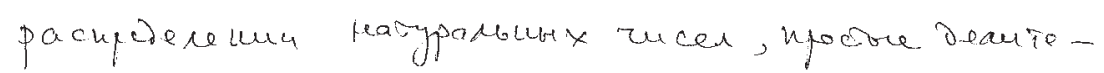

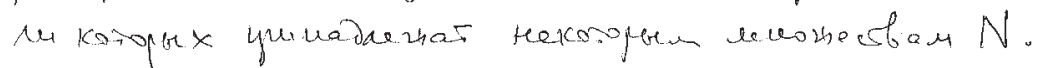

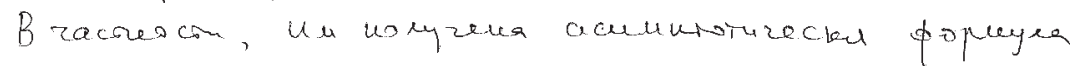
ane $\pi_{1}(x)$ - komroshe paryplabux recer, the ye-

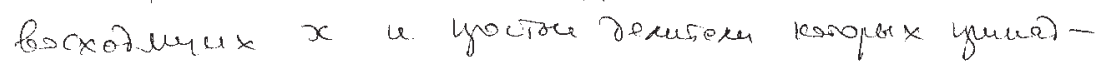

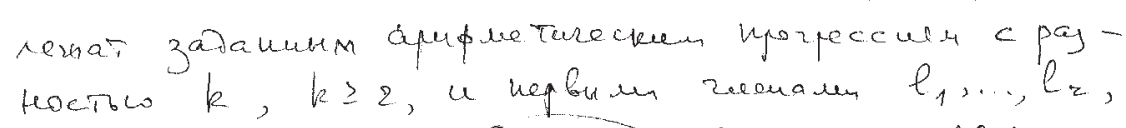
to $\left.1 \leq l<\ldots<l_{r}, r \leq \varphi(k)\right)\left(l_{r}, k\right)=1 ;=\left(l_{r}, k\right)=1$. $7_{\text {Ta }}$ popugie uner fou:

$$
\pi_{1}(x)=
$$

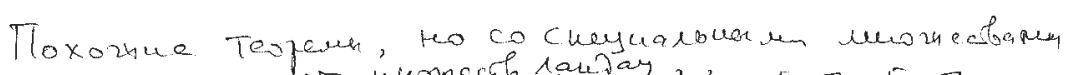

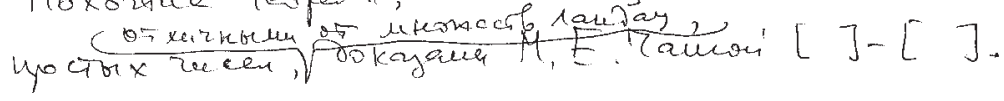

Ananuтuzeckü annapas dor dokazarenciba terpeub. Uueen pabeuciba:

$$
\frac{\zeta(2 s)}{\zeta(s)}=\sum_{n=1}^{\infty} \frac{\lambda(n)}{n^{s}},
$$

$$
* * *
$$

Cuedrateron,

$$
\begin{aligned}
& \sum_{u \leq x} b(n)=n_{0}^{*}(x)-n_{i}^{*}(x) \\
& \text { Kpove toi- }, \\
& n_{0}^{*}(x)+u_{i}^{*}(x)=n^{*}(x) .
\end{aligned}
$$

Рис. 1. Фрагменты рукописи А. А. Карацубы 
4. Эффект Чанги. Э. Ландау [1], [2] доказал также ряд общих теорем о распределении натуральных чисел, все простые делители которых принадлежат некоторым подмножествам $\mathbb{N}$. В частности, им получена асимптотическая формула для $n(x)$ - количества чисел $n$, не превосходящих $x$, все простые делители которых принадлежат заданным арифметическим прогрессиям с разностью $k, k \geqslant 2$, и первыми членами $l_{1}, \ldots, l_{r}$, где $1 \leqslant l_{1}<\cdots<l_{r}<k, r \leqslant \varphi(k)$, $\left(l_{1}, k\right)=1, \ldots,\left(l_{r}, k\right)=1$ (множество всех таких чисел обозначим через $\left.\mathbb{M}\right)$. Эта формула имеет вид:

$$
n(x)=c_{0} x(\ln x)^{r / \varphi(k)-1}+O\left(x(\ln x)^{r / \varphi(k)-2}\right) .
$$

Похожие теоремы, но со специальными множествами простых чисел, отличными от множеств Ландау, доказаны М. Е. Чангой в [4]-[6]. В этих работах получены также асимптотики сумм мультипликативных функций, причем суммирования ведутся по специальным множествам натуральных чисел, включая вышеуказанные. Здесь обнаруживается эффект поведения главного члена таких формул. В частности, если рассматривать сумму значений функции Мёбиуса, то во многих случаях при суммировании по специальным множествам эта сумма имеет явный асимптотический характер. Например, при $r<\varphi(k)$

$$
\sum_{n \leqslant x, n \in \mathbb{M}} \mu(n) \sim-c_{1} x(\ln x)^{-r / \varphi(k)-1}, \quad x \rightarrow+\infty,
$$

где $c_{1}>0$. При суммировании же по всем натуральным числам значений $\mu(n)$, как известно (см., например, [2] или [7]),

$$
\sum_{n \leqslant x} \mu(n)=O\left(x e^{-c \sqrt{\ln x}}\right), \quad x \rightarrow+\infty .
$$

Разница в поведении сумм функций Мёбиуса при суммировании по всему множеству натуральных чисел и при суммировании по множеству $\mathbb{M}$ назовем эффектом Чанги.

5. Основные результаты. Пусть $k \geqslant 2$ - натуральное число и заданы $r$ различных натуральных чисел $l_{1}, \ldots, l_{r}$ таких, что $1 \leqslant l_{j}<k,\left(l_{j}, k\right)=1$, $j=1, \ldots, r$.

Пусть $\mathbb{A}$ - множество простых чисел, принадлежащих прогрессиям $\mathrm{km}+\mathrm{l}_{j}$, $j \leqslant r$. Тривиальные случаи, когда $r=0(\mathbb{A}=\varnothing)$ и $r=\varphi(k)$ (A есть все простые числа, не делящие $k$ ), рассматривать не будем и далее предполагаем, что

$$
1 \leqslant r<\varphi(k) .
$$

Обозначим через $\mathbb{N}^{*}$ множество натуральных чисел, которые не имеют простых делителей из $\mathbb{A}$, через $\mathbb{N}_{0}^{*}$ подмножество чисел из $\mathbb{N}^{*}$ с четным числом простых делителей, через $\mathbb{N}_{1}^{*}$ подмножество чисел из $\mathbb{N}^{*}$ с нечетным числом простых делителей. Определим функции $n^{*}(x), n_{0}^{*}(x), n_{1}^{*}(x)$ равенствами

$$
n^{*}(x)=\sum_{n \leqslant x, n \in \mathbb{N}^{*}} 1, \quad n_{0}^{*}(x)=\sum_{n \leqslant x, n \in \mathbb{N}_{0}^{*}} 1, \quad n_{1}^{*}(x)=\sum_{n \leqslant x, n \in \mathbb{N}_{1}^{*}} 1 .
$$


Очевидно, что

$$
n_{0}^{*}(x)+n_{1}^{*}(x)=n^{*}(x)=\sum_{n \leqslant x} a(n),
$$

где $a(n)$ - характеристическая функция $\mathbb{N}^{*}$, и, кроме того,

$$
n_{0}^{*}(x)-n_{1}^{*}(x)=\sum_{n \leqslant x, n \in \mathbb{N}^{*}} \lambda(n)=\sum_{n \leqslant x} b(n),
$$

где $b(n)=\lambda(n) a(n)$ и $\lambda(n)=(-1)^{\alpha_{1}+\cdots+\alpha_{\nu}}$ при $n=p_{1}^{\alpha_{1}} \cdots p_{\nu}^{\alpha_{\nu}}$.

Теорема 1. При $x \rightarrow+\infty$ справедлива асимптотическая формула

$$
n_{1}^{*}(x)-n_{0}^{*}(x) \sim C n^{*}(x)(\ln x)^{2(r / \varphi(k)-1)},
$$

где $C$ - положительная постоянная.

Вместо введенного множества $\mathbb{N}^{*}$ можно рассматривать множество $\mathbb{N}^{* *} \subset \mathbb{N}^{*}$ такое, что $n \in \mathbb{N}^{* *}$, если $n \in \mathbb{N}^{*}$ и к тому же $n$ - бесквадратное число. Определяя $\mathbb{N}_{0}^{* *}$ и $\mathbb{N}_{1}^{* *}$ как подмножества $\mathbb{N}^{* *}$, содержащие числа с четным числом простых делителей и с нечетным числом простых делителей соответственно, а также функции $n^{* *}(x), n_{0}^{* *}(x), n_{1}^{* *}(x)$ как

$$
n^{* *}(x)=\sum_{n \leqslant x, n \in \mathbb{N}^{* *}} 1, \quad n_{0}^{* *}(x)=\sum_{n \leqslant x, n \in \mathbb{N}_{0}^{* *}} 1, \quad n_{1}^{* *}(x)=\sum_{n \leqslant x, n \in \mathbb{N}_{1}^{* *}} 1,
$$

можно доказать аналог теоремы 1.

Теорема 2. При $x \rightarrow+\infty$ справедлива асимптотическая формула

$$
n_{1}^{* *}(x)-n_{0}^{* *}(x) \sim C^{*} n^{* *}(x)(\ln x)^{2(r / \varphi(k)-1)},
$$

где $C^{*}$ - положительная постоянная.

Аналогичные теоремы можно доказать и для других множеств натуральных чисел, например для чисел, представимых в виде суммы двух квадратов (см. пример в п. 8). Очевидно также, что можно рассматривать множества натуральных чисел, у которых, напротив, все простые делители принадлежат $\mathbb{A}$, и доказать аналогичные теоремы, которые будут содержать те же асимптотики с точностью до констант.

Теперь рассмотрим нашу задачу в несколько более общей постановке. Пусть A - некоторое бесконечное множество простых чисел, $\pi(x ; \mathbf{A})$ - количество простых чисел множества $\mathbf{A}$, не превосходящих $x, \mathbf{N}^{*}-$ множество натуральных чисел $n$, которые не имеют делителей из $\mathbf{A}$, т. е. если $p$ - делитель $n$, то $p \notin \mathbf{A} ; \mathbf{N}_{0}^{*}$ и $\mathbf{N}_{1}^{*}$ - подмножества $\mathbf{N}^{*}$, причем $n \in \mathbf{N}_{0}^{*}$ тогда и только тогда, когда $n$ имеет четное количество простых делителей, $n \in \mathbf{N}_{1}^{*}$ тогда и только тогда, когда $n$ имеет нечетное количество простых делителей; $\mathbf{n}^{*}(x), \mathbf{n}_{0}^{*}(x)$, $\mathbf{n}_{1}^{*}(x)$ определяются равенствами

$$
\mathbf{n}^{*}(x)=\sum_{n \leqslant x, n \in \mathbf{N}^{*}} 1, \quad \mathbf{n}_{0}^{*}(x)=\sum_{n \leqslant x, n \in \mathbf{N}_{0}^{*}} 1, \quad \mathbf{n}_{1}^{*}(x)=\sum_{n \leqslant x, n \in \mathbf{N}_{1}^{*}} 1 .
$$


Предполагаем также, что

$$
\pi(x ; \mathbf{A})=\sum_{p \leqslant x, p \in \mathbf{A}} 1=C(\mathbf{A}) \pi(x)+r_{\mathbf{A}}(x),
$$

где $0<C(\mathbf{A})<1, r_{\mathbf{A}}(x)=O\left(x^{1-\Delta}\right), \Delta>0$. Имеет место следующая теорема.

ТеОРема 3. При $x \rightarrow+\infty$ справедлива асимптотическая формула

$$
\mathbf{n}_{1}^{*}(x)-\mathbf{n}_{0}^{*}(x) \sim C_{0}(\mathbf{A}) \mathbf{n}^{*}(x)(\ln x)^{2(C(\mathbf{A})-1)},
$$

где $C_{0}(\mathbf{A})$ - положительная постоянная.

Если применить теорему 3 к множеству А из [4]:

$$
\mathbf{A}=\bigcup_{n=0}^{\infty}\left[(D n+l-1)^{1 / a},(D n+l)^{1 / a}\right),
$$

где $a$ - нецелое положительное число, $D, l$ - натуральные числа, $D \geqslant 2,1 \leqslant$ $l \leqslant D$, то в этом случае формула $(12)$ имеет место с $C(\mathbf{A})=1 / D$.

Можно рассмотреть другой пример: представить все простые числа единым списком, занумеровав их подряд в порядке возрастания, и в качестве множества $\mathbf{A}$ взять каждое $k$-е простое число из списка $(k \geqslant 2)$. В этом случае в $(12)$ $C(\mathbf{A})=1 / k$

6. Доказательство основной теоремы 1. Вычислим асимптотики сумм $\sum_{n \leqslant x} a(n)$ и $\sum_{n \leqslant x} b(n)$ методом комплексного интегрирования. При этом опустим некоторые промежуточные этапы, подробное изложение которых является стандартным и может быть найдено в работе [6]. При $\operatorname{Re} s>1$ определим для каждого $l_{j}$ функцию

$$
F_{l_{j}}(s)=\prod_{p \equiv l_{j}(\bmod k)}\left(1-\frac{1}{p^{s}}\right)^{-1} .
$$

Известно, что эта функция может быть аналитически продолжена левее единичной прямой и для этой функции в области $\sigma \geqslant \beta=1-c_{1} / \ln T,|t| \leqslant T$, $T \geqslant 10$, разрезанной вдоль горизонтального отрезка, имеющего правым концом точку $s=1$, справедливо равенство

$$
F_{l_{j}}(s)=\sqrt[\varphi(k)]{\zeta(s)} e^{G_{l_{j}}(s)}
$$

где функция

$$
\begin{aligned}
G_{l_{j}}(s)= & \frac{1}{\varphi(k)} \sum_{\chi \neq \chi_{0}} \chi\left(l_{j}^{*}\right) \sum_{p} \sum_{m=2}^{\infty} \frac{\chi(p)-\chi^{m}(p)}{m p^{m s}} \\
& +\frac{1}{\varphi(k)} \sum_{p \mid k} \ln \left(1-\frac{1}{p^{s}}\right)+\frac{1}{\varphi(k)} \sum_{\chi \neq \chi_{0}} \chi\left(l_{j}^{*}\right) \ln L(s, \chi),
\end{aligned}
$$


$l_{j} l_{j}^{*} \equiv 1(\bmod k)$, аналитична в свободной от нулей $\zeta(s)$ области $\sigma \geqslant \beta=1-$ $c_{1} / \ln T,|t| \leqslant T$, а на границе этой области имеет место оценка

$$
\ln ^{-c_{2}} T \ll\left|F_{l_{j}}(s)\right| \ll \ln ^{c_{2}} T .
$$

При $\operatorname{Re} s>1$ имеем

$$
\begin{aligned}
& \zeta_{1}(s)=\sum_{n=1}^{\infty} \frac{a(n)}{n^{s}}=\zeta(s) \prod_{p \in \mathbb{A}}\left(1-\frac{1}{p^{s}}\right)=\zeta(s) \prod_{j=1}^{r} F_{l_{j}}^{-1}(s), \\
& F(s)=\sum_{n=1}^{\infty} \frac{b(n)}{n^{s}}=\frac{\zeta_{1}(2 s)}{\zeta_{1}(s)}=\frac{\zeta(2 s)}{\zeta(s)} \prod_{j=1}^{r} F_{l_{j}}(s) F_{l_{j}}^{-1}(2 s) .
\end{aligned}
$$

Применим к функциям $\zeta_{1}(s)$ и $F(s)$ формулу Перрона (см., например, [8]-[10]). Пусть $f(s)=\sum_{n=1}^{\infty} \frac{a_{n}}{n^{s}}$, причем $\sum_{n=1}^{\infty}\left|a_{n}\right| n^{-\sigma}<\infty$ при всех $\sigma>1$. Предположим, что $\left|a_{n}\right| \leqslant A(n)$, где $A(n)>0$ - монотонно возрастающая функция переменной $n$ и при $\sigma \rightarrow 1+0$ выполняется соотношение

$$
\sum_{n=1}^{\infty}\left|a_{n}\right| n^{-\sigma}=O\left((\sigma-1)^{-\alpha}\right)
$$

где $\alpha$ - некоторая положительная постоянная. Тогда при любых $b_{0} \geqslant b>1$, $T \geqslant 2, x \geqslant 2$ имеет место формула Перрона:

$$
\Phi(x)=\sum_{n \leqslant x} a_{n}=\frac{1}{2 \pi i} \int_{b-i T}^{b+i T} f(s) \frac{x^{s}}{s} d s+R(x),
$$

где

$$
R(x)=O\left(\frac{x^{b}}{T(b-1)^{\alpha}}+A(2 x)\left(\frac{x \ln x}{T}+\ln T\right)\right)
$$

и постоянная в знаке $O$ зависит только от $b_{0}$.

Для функций $\zeta_{1}(s)$ и $F(s)$ можно считать, что $A(n)=1$ и $\alpha=1$. Применяя формулу Перрона и выбирая в качестве параметров $b$ и $T$ для обеих рассматриваемых функций $b=1+1 / \ln x, T=e^{c_{1} \sqrt{\ln x}}$, получаем

$$
\begin{aligned}
& \sum_{n \leqslant x} a(n)=\frac{1}{2 \pi i} \int_{b-i T}^{b+i T} \zeta_{1}(s) \frac{x^{s}}{s} d s+O\left(x e^{-c_{3} \sqrt{\ln x}}\right), \\
& \sum_{n \leqslant x} b(n)=\frac{1}{2 \pi i} \int_{b-i T}^{b+i T} F(s) \frac{x^{s}}{s} d s+O\left(x e^{-c_{4} \sqrt{\ln x}}\right) .
\end{aligned}
$$

Функции $\zeta_{1}(s)$ и $F(s)$ аналитически продолжаются левее единичной прямой с помощью формулы (14):

$$
\begin{aligned}
\zeta_{1}(s)= & (\zeta(s))^{1-r / \varphi(k)} \exp \left(-G_{l_{1}}(s)-\cdots-G_{l_{r}}(s)\right) \\
F(s)= & (\zeta(s))^{r / \varphi(k)-1}(\zeta(2 s))^{1-r / \varphi(k)} \\
& \quad \times \exp \left(G_{l_{1}}(s)+\cdots+G_{l_{r}}(s)-G_{l_{1}}(2 s)-\cdots-G_{l_{r}}(2 s)\right) .
\end{aligned}
$$


Перепишем (22), (23) в виде

$$
\begin{aligned}
\frac{\zeta_{1}(s)}{s} & =(s-1)^{r / \varphi(k)-1} H_{a}(s), \\
\frac{F(s)}{s} & =(s-1)^{1-r / \varphi(k)} H_{b}(s),
\end{aligned}
$$

где

$$
\begin{aligned}
H_{a}(s)= & \frac{1}{s}((s-1) \zeta(s))^{1-r / \varphi(k)} \exp \left(-G_{l_{1}}(s)-\cdots-G_{l_{r}}(s)\right) \\
H_{b}(s)=\frac{1}{s} & ((s-1) \zeta(s))^{r / \varphi(k)-1}(\zeta(2 s))^{1-r / \varphi(k)} \\
& \quad \times \exp \left(G_{l_{1}}(s)+\cdots+G_{l_{r}}(s)-G_{l_{1}}(2 s)-\cdots-G_{l_{r}}(2 s)\right) .
\end{aligned}
$$

Далее, для интегралов из правых частей (20), (21) рассмотрим область, ограниченную контуром $C$, состоящим из отрезков, соединяющих последовательно точки $b-i T, \beta-i T$ и $\beta-i 0$, петли $\Upsilon$, обходящей точку $s=1$ против часовой стрелки, и отрезков, последовательно соединяющих точки $\beta+i 0, \beta+i T, b+i T$ и $b-i T$. Подынтегральная функция аналитична в замыкании этой области, следовательно, в силу интегральной теоремы Коши соответствующие интегралы по границе этой области равны 0, откуда получаем

$$
\begin{aligned}
& \frac{1}{2 \pi i} \int_{b-i T}^{b+i T} \zeta_{1}(s) \frac{x^{s}}{s} d s=\frac{1}{2 \pi i} \int_{\Upsilon} \zeta_{1}(s) \frac{x^{s}}{s} d s+O\left(x e^{-c_{5} \sqrt{\ln x}}\right), \\
& \frac{1}{2 \pi i} \int_{b-i T}^{b+i T} F(s) \frac{x^{s}}{s} d s=\frac{1}{2 \pi i} \int_{\Upsilon} F(s) \frac{x^{s}}{s} d s+O\left(x e^{-c_{6} \sqrt{\ln x}}\right) .
\end{aligned}
$$

При этом интегралы по контуру $C$ (исключая $\Upsilon$ и отрезок, соединяющий точки $b-i T$ и $b+i T)$ мы оценили тривиально, пользуясь верхней и нижней оценками для $\left|F_{l_{j}}(s)\right|$. Подставляя в (28), (29) функции $(24),(25)$, находим

$$
\frac{1}{2 \pi i} \int_{\Upsilon} \zeta_{1}(s) \frac{x^{s}}{s} d s=x I_{1}(x), \quad \frac{1}{2 \pi i} \int_{\Upsilon} F(s) \frac{x^{s}}{s} d s=x I_{2}(x),
$$

где

$$
\begin{aligned}
& I_{1}(x)=\frac{1}{2 \pi i} \int_{\Upsilon^{\prime}} x^{s} s^{r / \varphi(k)-1} H_{a}(s+1) d s, \\
& I_{2}(x)=\frac{1}{2 \pi i} \int_{\Upsilon^{\prime}} x^{s} s^{1-r / \varphi(k)} H_{b}(s+1) d s
\end{aligned}
$$

и функции $H_{a}(s), H_{b}(s)$ определяются формулами $(26),(27)$, а $\Upsilon^{\prime}$ - контур, полученный из $\Upsilon$ параллельным переносом на единицу влево вдоль вещественной оси, что соответствует замене $s$ на $s+1$.

Далее, для того чтобы выписать асимптотики сумм $\sum_{n \leqslant x} a(n)$ и $\sum_{n \leqslant x} b(n)$, воспользуемся следующим (доказанным в [6; лемма 2]) утверждением.

Пусть функция $H(s)$ аналитична в круге $|s|<\Delta$ и через $H_{j}$ обозначен $j$-й коэффициент ее разложения в ряд Тейлора в этом круге. Пусть контур $\Upsilon$ 
начинается в точке $-(\ln x)^{-1 / 2}-i 0$, обходит начало координат против часовой стрелки и заканчивается в точке $-(\ln x)^{-1 / 2}+i 0$. Пусть

$$
I(x)=\frac{1}{2 \pi i} \int_{\Upsilon} x^{s} s^{-\varrho / \varphi(k)} H(s) d s,
$$

где $\varrho$ - фиксированное целое число. Тогда имеет место асимптотическая формула

$$
I(x)=\sum_{0 \leqslant j \leqslant \sqrt{\ln x}} H_{j} \frac{(\ln x)^{\varrho / \varphi(k)-j-1}}{\Gamma(\varrho / \varphi(k)-j)}+O\left(e^{-C_{1} \sqrt{\ln x}}\right) .
$$

Кроме того, в (34) можно заменить верхний предел суммирования фиксированным целым неотрицательным $N$, причем остаток оценится величиной первого отброшенного члена, т. е. $O\left((\ln x)^{\varrho / \varphi(k)-N-2}\right)$.

Очевидно, что интегралы (31), (32) представляют собой интеграл вида (33), при этом $\varrho=\varphi(k)-r>0$ для $I_{1}(x)$ и $\varrho=r-\varphi(k)<0$ для $I_{2}(x)$ (мы рассматриваем только нетривиальные случаи, когда $0<r<\varphi(k))$. Из (31)-(34) получаем

$$
\begin{aligned}
& I_{1}(x)=\sum_{0 \leqslant j \leqslant \sqrt{\ln x}} A_{j} \frac{(\ln x)^{-r / \varphi(k)-j}}{\Gamma(1-r / \varphi(k)-j)}+O\left(e^{-C_{2} \sqrt{\ln x}}\right), \\
& I_{2}(x)=\sum_{0 \leqslant j \leqslant \sqrt{\ln x}} B_{j} \frac{(\ln x)^{r / \varphi(k)-j-2}}{\Gamma(r / \varphi(k)-j-1)}+O\left(e^{-C_{3} \sqrt{\ln x}}\right),
\end{aligned}
$$

где $A_{j}, B_{j}$ - коэффициенты разложения соответственно функций $H_{a}(s)$ и $H_{b}(s)$ в ряд Тейлора в точке $s=1$, при этом

$$
\begin{aligned}
& A_{0}=H_{a}(1)=\exp \left(-G_{l_{1}}(1)-\cdots-G_{l_{r}}(1)\right)>0, \\
& B_{0}=H_{b}(1)=\left(\frac{\pi^{2}}{6}\right)^{1-r / \varphi(k)} \\
& \quad \quad \times \exp \left(G_{l_{1}}(1)+\cdots+G_{l_{r}}(1)-G_{l_{1}}(2)-\cdots-G_{l_{r}}(2)\right)>0 .
\end{aligned}
$$

Обозначим

$$
a_{j}=\frac{A_{j}}{\Gamma(1-r / \varphi(k)-j)}, \quad b_{j}=\frac{B_{j}}{\Gamma(r / \varphi(k)-1-j)} .
$$

Из (20), (21), (28)-(30) и (35)-(39) находим

$$
\begin{aligned}
& \sum_{n \leqslant x} a(n)= a_{0} x(\ln x)^{-r / \varphi(k)}+a_{1} x(\ln x)^{-r / \varphi(k)-1} \\
&+O\left(x(\ln x)^{-r / \varphi(k)-2}\right), \quad x \rightarrow+\infty, \\
& \sum_{n \leqslant x} b(n)=b_{0} x(\ln x)^{r / \varphi(k)-2}+O\left(x(\ln x)^{r / \varphi(k)-3}\right), \quad x \rightarrow+\infty .
\end{aligned}
$$

Рассмотрим асимптотики (40), (41). Заметим, что, поскольку $0<r / \varphi(k)<1$, из $(39)$ следует, что $a_{0}>0, b_{0}<0$. Поскольку

$$
-\frac{r}{\varphi(k)}>\frac{r}{\varphi(k)}-2, \quad \frac{r}{\varphi(k)}-2>-\frac{r}{\varphi(k)}-2, \quad-\frac{r}{\varphi(k)}-1>\frac{r}{\varphi(k)}-3,
$$


то, учитывая (9), (10), получаем асимптотики для величин $n_{0}^{*}(x), n_{1}^{*}(x)$ :

$$
\begin{array}{rl}
n_{0}^{*}(x)=h_{0} & x(\ln x)^{-r / \varphi(k)}+h_{1} x(\ln x)^{-r / \varphi(k)-1}-h x(\ln x)^{r / \varphi(k)-2} \\
& +O\left(x(\ln x)^{-r / \varphi(k)-2}+x(\ln x)^{r / \varphi(k)-3}\right), \quad x \rightarrow+\infty, \\
n_{1}^{*}(x)=h_{0} x(\ln x)^{-r / \varphi(k)}+h_{1} x(\ln x)^{-r / \varphi(k)-1}+h x(\ln x)^{r / \varphi(k)-2} \\
+ & O\left(x(\ln x)^{-r / \varphi(k)-2}+x(\ln x)^{r / \varphi(k)-3}\right), \quad x \rightarrow+\infty,
\end{array}
$$

где

$$
\begin{gathered}
h_{0}=h_{0}\left(r, k, l_{1}, \ldots, l_{r}\right)=\frac{a_{0}}{2}>0, \quad h_{1}=h_{1}\left(r, k, l_{1}, \ldots, l_{r}\right)=\frac{a_{1}}{2}, \\
h=h\left(r, k, l_{1}, \ldots, l_{r}\right)=-\frac{b_{0}}{2}>0 .
\end{gathered}
$$

Легко видеть, что из асимптотик (43), (44) следует, что

$$
n_{1}^{*}(x)-n_{0}^{*}(x) \sim 2 h x(\ln x)^{r / \varphi(k)-2} \sim \frac{h}{h_{0}} n^{*}(x)(\ln x)^{2(r / \varphi(k)-1)}, \quad x \rightarrow+\infty .
$$

Теорема доказана.

\section{7. Замечания к доказательству теоремы 1 .}

ЗАмЕчАниЕ 1 . В то время как из теоремы 1 следует, что

$$
n_{1}^{*}(x)-n_{0}^{*}(x) \rightarrow+\infty, \quad x \rightarrow+\infty,
$$

из асимптотик $(43),(44)$ для величин $n_{1}^{*}(x), n_{0}^{*}(x)$ находим

$$
n_{0}^{*}(x) \sim n_{1}^{*}(x) \sim \frac{1}{2} n^{*}(x) \sim h_{0} x(\ln x)^{-r / \varphi(k)}, \quad x \rightarrow+\infty,
$$

т. е. чисел в множествах $\mathbb{N}_{0}^{*}$ и $\mathbb{N}_{1}^{*}$ асимптотически поровну, что аналогично результату Ландау (3).

ЗАмечАние 2. Теорема 2 доказывается аналогично теореме 1 с точностью до констант, при этом в качестве функции (16) участвует функция

$$
\sum_{n=1}^{\infty} \frac{a_{1}(n)}{n^{s}}=\frac{\zeta_{1}(s)}{\zeta_{1}(2 s)}=\frac{\zeta(s)}{\zeta(2 s)} \prod_{j=1}^{r} F_{l_{j}}^{-1}(s) F_{l_{j}}(2 s),
$$

а в качестве функции (17) - функция

$$
\sum_{n=1}^{\infty} \frac{b_{1}(n)}{n^{s}}=\prod_{j=1}^{r} F_{l_{j}}^{-1}(s)
$$

ЗАмЕчАниЕ 3. При доказательстве теоремы 3 в случае произвольного множества А используются функции

$$
\begin{aligned}
& \zeta_{1}(s)=\zeta(s) \prod_{p \in \mathbf{A}}\left(1-\frac{1}{p^{s}}\right)=(\zeta(s))^{1-C(\mathbf{A})} e^{-G(s)}, \\
& F(s)=(\zeta(s))^{C(\mathbf{A})-1}(\zeta(2 s))^{1-C(\mathbf{A})} e^{G(s)-G(2 s)} .
\end{aligned}
$$


Доказательство проводится аналогично доказательству теоремы 1, но с другими константами и с заменой $r / \varphi(k)$ на $C(\mathbf{A})$. Аналитическое продолжение получается с помощью преобразования Абеля, при этом

$$
G(s)=s \int_{2}^{\infty} \frac{r_{\mathbf{A}}(u) d u}{u\left(u^{s}-1\right)} .
$$

8. Пример. Числа, представимые суммой двух квадратов. Приведем наглядный пример проявления описанного эффекта. Пусть $\widetilde{\mathbb{N}}-$ множество натуральных чисел, представимых суммой двух квадратов целых чисел, $\widetilde{\mathbb{N}}_{0}$ - подмножество $\widetilde{\mathbb{N}}$, включающее числа с четным количеством простых сомножителей, $\widetilde{\mathbb{N}}_{1}-$ подмножество $\widetilde{\mathbb{N}}$, состоящее из чисел с нечетным количеством простых сомножителей. Как и выше, определим следующие функции:

$$
\widetilde{n}(x)=\sum_{n \leqslant x, n \in \widetilde{\mathbb{N}}} 1, \quad \widetilde{n}_{0}(x)=\sum_{n \leqslant x, n \in \widetilde{\mathbb{N}}_{0}} 1, \quad \widetilde{n}_{1}(x)=\sum_{n \leqslant x, n \in \widetilde{\mathbb{N}}_{1}} 1,
$$

Известно, что натуральное число представимо суммой двух квадратов в том и только том случае, если все простые вида $4 n+3$ входят в его каноническое разложение в четных степенях. Возьмем $k=4$ и повторим доказательство теоремы 1 с функцией

$$
\widetilde{\zeta}_{1}(s)=\prod_{p \neq \equiv 3(\bmod 4)}\left(1-\frac{1}{p^{s}}\right)^{-1} \prod_{p \equiv 3(\bmod 4)}\left(1-\frac{1}{p^{2 s}}\right)^{-1}=\zeta(s) F_{3}^{-1}(s) F_{3}(2 s)
$$

в качестве функции (16) и с функцией

$$
\widetilde{F}(s)=\prod_{p \neq \equiv 3(\bmod 4)}\left(1+\frac{1}{p^{s}}\right)^{-1} \prod_{p \equiv 3(\bmod 4)}\left(1-\frac{1}{p^{2 s}}\right)^{-1}=\frac{\zeta(2 s)}{\zeta(s)} F_{3}(s)
$$

в качестве функции (17). Получим при $x \rightarrow+\infty$

$$
\begin{aligned}
\widetilde{n}(x) & \sim \frac{1}{\sqrt{\pi}} F_{3}(2) e^{-G_{3}(1)} \frac{x}{\sqrt{\ln x}}, \\
\widetilde{n}_{1}(x)-\widetilde{n}_{0}(x) & \sim \frac{1}{2 \sqrt{\pi}} \zeta(2) e^{G_{3}(1)} \frac{x}{(\sqrt{\ln x})^{3}} .
\end{aligned}
$$

При $k=4$ формула (15) дает

$$
e^{G_{3}(1)}=\sqrt{\frac{2}{\pi}} \prod_{p \equiv 3(\bmod 4)}\left(1-\frac{1}{p^{2}}\right)^{-1 / 2}=\sqrt{\frac{2 F_{3}(2)}{\pi}} .
$$

Следовательно,

$$
\widetilde{n}_{1}(x)-\widetilde{n}_{0}(x) \sim \frac{\pi}{6} \frac{\widetilde{n}(x)}{\ln x} \quad \text { при } x \rightarrow+\infty .
$$

\section{Список литературы}

[1] E. Landau, "Über die Anzahl der Gitterpunkte in gewissen Bereichen", Gött. Nachr., 1912, 687-770.

[2] E. Landau, Handbuch der Lehre von der Verteilung der Primzahlen, 2, Teubner, Leipzig, 1909. 
[3] Ло-Кен Хуа, Метод тригонометрических сумм и его применение в теории чисел, Мир, М., 1964, 188 с.; пер. с нем.: Loo-Keng Hua, Die Abschätzung von Exponentialsummen und ihre Anwendung in der Zahlentheorie, Enzyklopädie Math. Wiss., I, Teubner, Leipzig, 1959, 123 pp.

[4] М.Е. Чанга, "О числах, все простые делители которых лежат в специальных промежутках", Изв. РАН. Сер. матем., 67:4 (2003), 213-224; англ. пер.: M. E. Changa, "Numbers whose prime divisors lie in special intervals", Izv. Math., 67:4 (2003), 837-848.

[5] М.Е. Чанга, Арифметические задачи с числами, все простые делители которых принадлежат специалъным множествам, Дис. ... докт. физ.-матем. наук, МИАН, М., 2004.

[6] М.Е. Чанга, "О суммах мультипликативных функций по числам, все простые делители которых принадлежат заданным арифметическим прогрессиям", Изв. РАН. Сер. матем., 69:2 (2005), 205-220; англ. пер.: М. Е. Changa, "On sums of multiplicative functions over numbers all of whose prime divisors belong to given arithmetic progressions", Izv. Math., 69:2 (2005), 423-438.

[7] К. Прахар, Распределение простьх чисел, Мир, М., 1967, 511 с.; пер. с нем.: K. Prachar, Primzahlverteilung, Springer-Verlag, Berlin-Göttingen-Heidelberg, 1957, $\mathrm{x}+415 \mathrm{pp}$.

[8] А. А. Карацуба, Основы аналитической теории чисел, Наука, М., 1975, 183 с.; англ. пер. 2-го изд.: А. А. Karatsuba, Basic analytic number theory, Springer-Verlag, Berlin, 1993, ISBN: 3-540-53345-1, xiv+222 pp.

[9] A. A. Karatsuba, Complex analysis in number theory, CRC Press, Boca Raton, FL, 1995, ISBN: 0-8493-2866-7, x+187 pp.

[10] С. М. Воронин, А.А. Карацуба, Дзета-функиия Римана, Физматлит, М., 1994, ISBN: 5-02-014120-8, 376 с.; англ. пер.: A. A. Karatsuba, S. M. Voronin, The Riemann zeta-function, de Gruyter Exp. Math., 5, Walter de Gruyter, Berlin, 1992, ISBN: 3-11-013170-6, xii+396 pp.

Поступила в редакцию 06.01 .2011

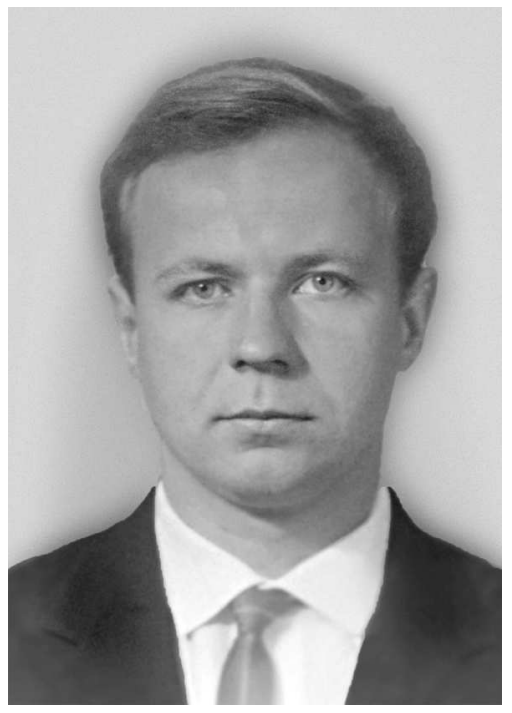

\section{От составителей статьи}

Анатолий Алексеевич Карацуба обнаружил интересный эффект: разность мощностей множеств чисел с нечетным и четным количеством простых сомножителей в случае, когда на эти сомножители накладываются некоторые ограничения, велика (стремится к бесконечности).

Предлагаем назвать обнаруженный эффект именем Анатолия Карацубы. 